\title{
Does it require to exclude cardiobiliary reflex in every acute coronary syndrome follow up patient with bedside ultrasound on emergency department
}

\author{
Mustafa Bolatkale', Çağdaş Can², Ahmet Çağdaş Acara ${ }^{3 *}$, Mustafa Topuz ${ }^{4}$ \\ 1 Medipol University Hospital, İstanbul, Turkey \\ ${ }^{2}$ Manisa State Hospital, Manisa, Turkey \\ ${ }^{3}$ Gaziemir State Hospital, İzmir, Turkey \\ ${ }^{4}$ Adana Numune Education and Research Hospital, Department of Cardiology, Adana, Turkey
}

\author{
Received: 14 June 2016 \\ Accepted: 18 July 2016 \\ Published online: 29 August 2016 \\ *Corresponding author: Ahmet \\ Çağdaş Acara; Gaziemir State Hospital, \\ İzmir, Turkey. \\ Email: cagdasacara@gmail.com \\ Competing interests: None. \\ Funding information: None. \\ Citation: Bolatkale M, Can Ç, Çağdaş \\ Acara A, Topuz M. Does it require to \\ exclude cardiobiliary reflex in every \\ acute coronary syndrome follow up \\ patient with bedside ultrasound on \\ emergency department. Journal of \\ Emergency Practice and Trauma 2017; \\ 3(2): 40-41. doi: $10.15171 /$ jept.2016.13
}

\begin{abstract}
In emergency department, physicians can diagnose pulseless electrical activity, asystole pericardial effusions, ischemic heart disease, wall motion abnormalities, valvular cardiac disease volume status or global cardiac function evaluating with electrocardiographic findings or using bedside cardiac ultrasonography. But these two methods are not always sufficient to explain the underlying another pathologies such as pancreatitis and acute cholecystitis which can mimick acute cardiac events. Patients who are followed up with a preliminary diagnosis of acute coronary syndrome in the emergency department, might have underlying biliary or pancreatic pathologies, or even more, these might be the sole reason of the clinical picture. So bedside abdomen ultrasonography and liver enzymes may be requested in all patients with suspected cardiac pathology with a normal cardiac ultrasonography when a patient presented with acute chest or abdominal pain. Physicians must be aware for coexisting pathophysiologies and take into account the differential diagnosis of all life-threatening causes such as cardiac ischemia or acute abdominal situations. So the diagnostic tests for gallbladder pathology could be added to cardiac ultrasonography.

Keywords: Cardiobiliary reflex, Acute coronary syndrome, Ultrasound
\end{abstract}

\section{Dear Editor}

In emergency department, physicians can diagnose pulseless electrical activity, asystole, pericardial effusions, ischemic heart disease, wall motion abnormalities, valvular cardiac disease volume status or global cardiac function. These can be achieved by electrocardiography (ECG) or bedside cardiac ultrasonography method. But these two methods are not always sufficient to explain the underlying pathologies which can mimick acute coronary syndrome (ACS) such as acute cholecystitis, biliary colic and acute pancreatitis.

Heart related diseases are not the unique reason of pathological ECG findings and elevated troponins. Patients with chest pain, ECG and cardiac troponins are two widely validated rules for diagnosing of ACS. In patients with normal ECG, cardiac troponins with high sensitivity and specificity can be applied to rule out ACS (1). The elevation of troponin levels in non-ischemic cardiac conditions may lead to a misclassification of patients. ACS is a potentially fatal condition and also anticoagulation is associated with the risk of major bleeding, so the false diagnosis can be mortal. The unnecessary thrombolytic treatment and invasive interventions had been previously reported in patients with cholecystitis and pancreatitis (2).

The signs and symptoms of gallbladder and heart disease may overlap, making diagnosis difficult. Patients with acute gallbladder disease or acute pancreatitis could have retrosternal pain, nausea and dyspnea resemble with ACS. On clinical examination, it may often be difficult to determine right upper quadrant tenderness from chest pain (3). In addition to clinical similarity, these pathologies can also mimick ACS in patients with normal coronary arteries in terms of cardiac biomarkers and ECG findings due to cardiobiliary reflex (1). A vagally mediated cardiobiliary reflex is the presumed cause of ECG abnormalities and increased cardiac troponin levels. It has been mentioned that acute cholecystitis might be the sole reason of ischemic ECG changes in a patient with no underlying cardiac disease (4). Also, it was shown that more than 50\% of the patients with acute pancreatitis had ECG abnormalities and some of these changes are suggesting an ACS. In acute pancreatitis, pancreatic proteolytic enzymes such as trypsin may have resulted in direct injury to the pericardium or myocyte membrane leading to changes in cell 
permeability with possible necrosis and electrical changes seen on ECG such as T wave inversion, ST segment depression or ST segment elevation $(5,6)$.

Both acute pancreatitis and acute cholecystitis with gallbladder distension are clinical disorders which could be a reason of the increased troponin I level $(2,5)$. Although elevated troponin levels in acute cholecystitis are mainly due to cardiobiliary reflex, myocardial stunning, a direct cardiac toxic effect by pancreatic proteolytic enzymes, electrolyte abnormalities and coronary vasospasm might be other reasons for elevation of cardiac biomarkers in acute pancreatitis (5). So we strongly believe that, patients who are followed up with a preliminary diagnosis of ACS in the emergency department, might have underlying biliary pathologies, or even more, this might be the one and only reason of the clinical picture. So beside abdomen ultrasonography and liver enzymes may be requested in all patients with suspected cardiac pathology with a normal cardiac ultrasonography.

In conclusion, when a patient presented to emergency department with acute chest or abdominal pain, the diagnostic tests for gallbladder pathology should be added to cardiac ultrasonography.

\section{Ethical issues}

Not applicable.

\section{Author's contribution}

All authors contributed to the intellectual planning of the project, intellectual analysis of the data, and writing of the paper.

\section{References}

1. Thygesen K, Alpert JS, White HD. Universal definition of myocardial infarction. Eur Heart J 2007; 28(20): 2525-38. doi: 10.1093/eurheartj/ehm355.

2. Demarchi MS, Regusci L, Fasolini F. Electrocardiographic changes and false-positive troponin I in a patient with acute cholecystitis. Case Rep Gastroenterol 2012; 6(2): 410-4. doi: 10.1159/000339965.

3. Husainy MA, Gopalan D, Pakkal M, Raj V. Mimics of acute coronary syndrome on MDCT. Emerg Radiol 2013; 20(3): 235-42. doi: 10.1007/s10140-012-1097-1.

4. Patel N, Ariyarathenam A, Davies W, Harris A. Acute cholecystits leading to ischemic ECG changes in a patient with no underlying cardiac disease. JSLS 2011; 15(1): 105-8. doi: 10.4293/108680811x13022985131534.

5. Kellner A, Robertson T. Selective necrosis of cardiac and skeletal muscle induced experimentally by means of proteolytic enzyme solutions given intravenously. J Exp Med 1954; 99(4): 387-404.

6. Cattermole GN, McKay N. Pseudo myocardial infarction. Emerg Med J 2006; 23(8): e48. doi: 10.1136/ emj.2005.032656. 